\title{
Recent Progress in Ferroelectric Diodes: Explora- tions in Switchable Diode Effect
}

\author{
Chen Ge, Can Wang, Kui-juan Jin*, Hui-bin Lu, Guo-zhen Yang
}

(Received 11 March 2013; accepted 17 April 2013; published online 28 April 2013)

\begin{abstract}
Switchable diode effect in ferroelectric diodes has attracted much attention for its potential applications in novel nonvolatile memories. We briefly review recent investigations on the switchable diode effect in ferroelectric diodes both experimentally and theoretically. Many recent studies demonstrate that the interfacial barrier between the metal-ferroelectrics could be modulated by the polarization charges, and the ferroelectric polarization that can be reversed by an external electric field plays a dominant role in the switchable diode effect. Moreover, we review a self-consistent numerical model, which can well describe the switchable diode effect in ferroelectric diodes. Based on this model, it can be predicted that it is a better choice to select metals with a smaller permittivity, such as noble metals, to obtain a more pronounced switchable diode effect in ferroelectric diodes.
\end{abstract}

Keywords: Ferroelectric diodes; Switchable diode effect; Metal/ferroelectrics/metal structure; Self-consistent mode

Citation: Chen Ge, Can Wang, Kui-juan Jin, Hui-bin Lu and Guo-zhen Yang, "Recent Progress in Ferroelectric Diodes: Explorations in Switchable Diode Effect", Nano-Micro Lett. 5(2), 81-87 (2013). http://dx. doi.org/10.5101/nml.v5i2.p81-87

\section{Introduction}

Ferroelectric materials are of characteristics to maintain the polarization state in the absence of a voltage and the possibility to reverse the polarization direction by applying an electric field $[1,2]$. It is an obvious idea to design robust nonvolatile random access memories based on intrinsic switchable ferroelectric polarization in the ferroelectric materials. Ferroelectric capacitive memories were regarded as a promising candidate for the next generation nonvolatile memories [3,4], but failed to achieve their commercial success mainly because of the scaling limit and the destructive readout that memories must revert to the original polarization state after reading the stored information [5]. It is of great value to explore an alternative route to nondestructively read the polarization in ferroelectric thin films.

A promising solution to these problems is to probe the polarization by measuring resistance states instead of sensing charges in metal/ferroelectrics/metal (MFM) structures [6]. The polarization charges can affect the barrier at the interface between electrodes and ferroelectrics, and the reversal of the polarization produces a change in the band diagram $[7,8]$. As a result of this, bistable resistance states can be obtained for two opposite polarizations. In other words, the high and low resistance states stem from the interplay of polarization and conduction. Two ferroelectric resistive switching concepts are classified by the conduction mechanism. In the case of ferroelectric ultrathin films, the current transport through ferroelectrics is governed by the tunneling process, which has emerged as an active research topic called as ferroelectric tunnel junctions [9-13]. In the case of ferroelectric thin films, the drift-diffusion or thermionic emission plays a dominant role in transport. The structures in the latter case are called as ferroelectric diodes, which is the focus of this review. For the

Beijing National Laboratory for Condensed Matter Physics, Institute of Physics, Chinese Academy of Sciences, Beijing 100190, China

*Corresponding author. E-mail: kjjin@iphy.ac.cn 
general hysteretic resistance concept, please refer to ref. [6].

Ferroelectric diodes have been investigated in the past two decades due to their huge potentials in nonvolatile memory applications. Blom et al. first reported bistable resistance states in 1994, showing that in the $\mathrm{La}_{0.5} \mathrm{Sr}_{0.5} \mathrm{CoO}_{3} / \mathrm{PbTiO}_{3} / \mathrm{Au}$ structure the conducting state exists under one polarization while the insulating state exists under the other polarization [14]. Since then many similar phenomena have been observed in various ferroelectric thin films [15-19]. Most interestingly, the switchable diode effect has been demonstrated recently in various ferroelectric structures ranging from organic films [20,21], inorganic bulks [22-24], and inorganic films [25-29]. The switchable diode effect means that the polarity of the diode can be reproducibly switched by the reversion of the applied electric field in the MFM structures, due to the polarization modulated barrier. In addition, a few theoretical studies on this issue have been presented to reveal the underlying physics in the switchable diode effect [30-32].

In this paper, we will briefly review some recent research progress in the switchable diode effect in ferroelectric diodes, and then discuss the experimental studies on the origin of the switchable diode effect and the role of oxygen vacancies. We will also briefly present a theoretical model, which can well describe the transport property in MFM structures. Before we conclude this article, a short discussion on the effect of electrodes will be presented.

\section{Recent progress in switchable ferroelec- tric diodes}

Up to now, the research on the switchable diode effect is mainly focused on $\mathrm{BiFeO}_{3}$ (BFO) materials [22,25-29]. In most cases, the BFO thin films were grown on the bottom electrode covered $\mathrm{SrTiO}_{3}$ substrates by pulsed laser deposition, and then the top electrodes were deposited on the BFO films to form MFM structures. $\mathrm{SrRuO}_{3}(\mathrm{SRO}) / \mathrm{BFO} / \mathrm{Pt}$ is a most common structure, in which an obvious current hysteresis behavior was observed as shown in Fig. 1(a) [27]. The measurements were repeatedly performed with 25 cycles by sweeping the bias voltage of the top electrode from 8 to $-7.5 \mathrm{~V}$ and back to $8 \mathrm{~V}$. The numbers in Fig. 1(a) denote the sequence of voltage sweeps. The $I-V$ curves show an obvious hysteresis behavior, and two $I-V$ segments in the range of $\pm 5 \mathrm{~V}$ are plotted using solid circle symbols and open circle symbols in the inset of Fig. 1(a) respectively, indicating a switchable diode effect. Figure 1(b) [27] shows the $I$ - $V$ curves plotted on semi-logarithmic coordinates for the 120 and $240 \mathrm{~nm}$ BFO films. The switchable diode effect was also observed for the $120 \mathrm{~nm}$ film, where the applied voltage range is smaller than that for the $240 \mathrm{~nm}$ film (about $\pm 3 \mathrm{~V})$. The resistance switching ratio is 103 at $-3 \mathrm{~V}$ for the $240 \mathrm{~nm}$ films, and it is 79 at $-1 \mathrm{~V}$ for the 120 nm films.

To further confirm the observed switchable diode effect, Wang et al. used electrical pulses of $\pm 8 \mathrm{~V}$ for $200 \mathrm{~ms}$ to induce the reversion of the polarization in the $240 \mathrm{~nm}$ BFO film, and measured $I-V$ curves with a sweep voltage of $\pm 2 \mathrm{~V}$ as shown in Fig. 2 [27,30]. Quite obviously, the structure shows a forward diode-like behavior after the electrical pulse of $+8 \mathrm{~V}$, whereas the structure shows a reverse diode-like behavior after the pulse of $-8 \mathrm{~V}$. It is worth mentioning that an excellent retention was obtained in this structure ascribed to
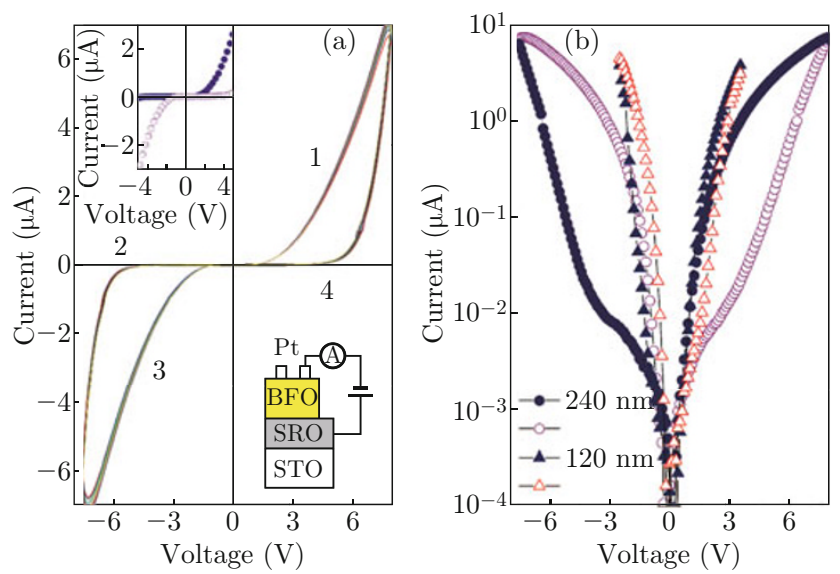

Fig. 1 (a) $I-V$ curves measured on a $240 \mathrm{~nm}$ BFO thin film; Insets show the sketch of the set up for the $I-V$ measurements and $I-V$ curves at the voltage range of $\pm 5 \mathrm{~V}$ which indicate the forward and reverse diode characteristics. (b) $I$ $V$ curves for the $240 \mathrm{~nm}$ and $120 \mathrm{~nm}$ BFO films plotted on semi-logarithmic scales. (Reprinted with permission from Ref. [27]. Copyright 2011, American Institute of Physics)

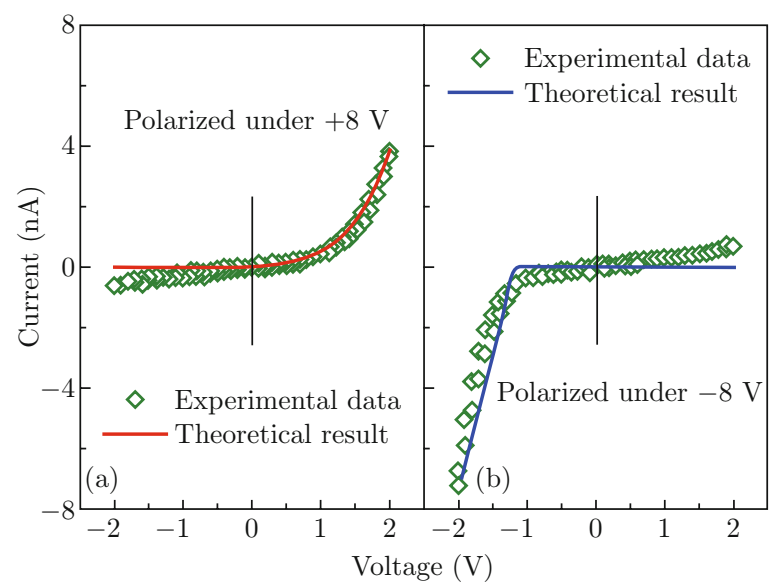

Fig. 2 The experimental (diamonds) and calculated (lines) $I-V$ curves in the case of right polarization (a) and left polarization (b) for the $\mathrm{SRO} / \mathrm{BFO} / \mathrm{Pt}$ structure. (Reprinted with permission from Ref. [30]. Copyright 2011, American Institute of Physics) 
the intrinsic non-volatile nature of spontaneous polarization [26].

An interesting question focuses on what is the origin of the switchable diode. Yang et al. presented the observation of the switchable diode effect in the Ca-doped BFO thin film [25]. It was hypothesized that the mobile oxygen vacancies created to maintain charge neutrality are the key to understand the transport property in this system. For one bias condition, oxygen vacancies can move and pile up near the top surface. Consequently, the donor oxygen vacancies and the acceptor calcium ions are locally unbalanced. Thus the top surface region becomes $n$-type, whereas the region near the bottom electrode becomes $p$-type. Then a diode can be formed. For the other bias condition, oxygen vacancies can move and pile up at the bottom surface, resulting in the inversion of the conduction direction in the diode.

However, many recent investigations have demonstrated a dominant role played by the ferroelectric polarization in switchable diode effect [26-29]. Jiang et al. confirmed that the resistive switching curves of the $\mathrm{SRO} / \mathrm{BFO} / \mathrm{Pt}$ structure have identical shapes to the Polarzation-Voltage loops. Combined the measurement results of the local polarization and diode current, they concluded that the switchable diode effect in ferroelectric diodes is completely governed by the ferroelectric polarization [26]. Moreover, Wang et al. measured the $I-V$ curves in the virgin $240 \mathrm{~nm}$ BFO film capacitance by increasing the sweep range step by step [27], to verify the correlation between the electrical hysteresis and ferroelectric polarization. Obvious hysteresis loops were not observed until the sweep voltages were larger than $\pm 6 \mathrm{~V}$ which is close to the coercive voltage of the ferroelectric thin film. The larger the voltage range, the more pronounced the hysteresis will become. It was claimed that the current hysteresis and diode-like behavior can be triggered and switched under a high enough electric field, which can drive the ferroelectric domain switching. Furthermore, Lee et al. also overlapped the response of the $J-V$ curves with the $P-V$ hysteresis loop [28]. Their results showed that the hysteresis behavior of the diode-polarity switching coincides with the ferroelectric switching hysteresis. They claimed that the sharp changes in current values near the coercive voltage exclude the possibility that the switchable diode originated from the migration of oxygen vacancies. The switch-ability between the reverse and forward diodes originates from the band modification and associated change in the barriers at the $\mathrm{BFO} /$ metal interfaces, which depends on the ferroelectric polarization direction. By using Conductive-AFM and PFM techniques, Hong et al. observed a switchable diode effect induced by the ferroelectric polarization in BFO nano-islands [29]. In short, most of recent experiments have proved that the polarization dominates the switchable diode effect in ferroelectric diodes.
The role of oxygen vacancy should be carefully considered, because the distribution of charged oxygen vacancy could affect charge transport in ferroelectric diodes. Lee et al. observed one-side diode effect in $\mathrm{SRO} / \mathrm{BFO} / \mathrm{Pt}$, and attributed this phenomenon to the interfacial defective layer possibly induced by the charged oxygen vacancies [28]. During the film deposition process, the downward self-polarization will cause the positively charged oxygen vacancies to migrate toward the top $\mathrm{Pt} / \mathrm{BFO}$ interface to compensate for the negative polarization charge [33], resulting in a defective layer. The defective layer can seriously affect charge transport at the $\mathrm{Pt} / \mathrm{BFO}$ interface, especially the carrier injection under a positive bias. In order to obtain a switchable diode effect, the defective layer induced by the oxygen vacancies should be removed from the samples. Lee et al. applied a positive dc bias of $+15 \mathrm{~V}$ to the as-grown sample for $30 \mathrm{~min}$ at room temperature. As a result, the transport behaviors between forward and reverse diodes in BFO thin film capacitors could be switched with the polarization reversal of the ferroelectric layer [28].

\section{Theoretical explanations}

For a deeper understanding of the switchable diode effect, it is fundamentally important to carry out a theoretical study on this issue. To that end, a key step is to construct a viable model to quantitatively calculate the band diagram, the charge distribution, and the transport property in MFM structures. One point that must be properly handled is that a ferroelectric thin film cannot be considered in isolation, because the measured property reflects the entire system of films, interfaces, and electrodes [2]. Recently, we have proposed a self-consistent model, particularly taking account of the incomplete screening effect of realistic metal electrodes $[34,35]$, to explain the switchable diode effect in MFM structures $[30,31]$. The fundamental charge transport equations in this system are as follows:

$$
\begin{gathered}
\frac{d^{2} \phi(x)}{d x^{2}}=-\frac{e}{\varepsilon(x)} \rho(x) \\
\frac{1}{e} \frac{d j(x)}{d x}-R(x)=0
\end{gathered}
$$

where $x$ is the spatial coordinate, and $e$ denotes the elementary charge. $\phi(x), \varepsilon(x), \rho(x), j(x)$, and $R(x)$ represent the electrostatic potential, dielectric constant, charge density, current density, recombination rate, respectively. The current density $j(x)$ and conductivity $\sigma(x)$ read

$$
\begin{aligned}
& j(x)=\frac{\sigma(x)}{e} \frac{d \kappa(x)}{d x} \\
& \sigma(x)=e \mu(x) n(x)
\end{aligned}
$$


where $\kappa(x), \mu(x)$, and $n(x)$ represent the electrochemical potential, mobility, and electron density, respectively. To write the electrochemical potential of metals $\kappa_{m}(x)$, the free electron model and Thomas-Fermi approximation are employed $[36,37]$. The semiconductor theory is utilized to calculate the electrochemical potential of ferroelectric films $\kappa_{s}(x)$. The electrochemical potential $\kappa_{m}(x)$ and $\kappa_{s}(x)$ are given by

$$
\begin{aligned}
& \kappa_{m}(x)=\frac{\hbar^{2}}{2 m}\left[3 \pi^{2} n_{m}(x)\right]^{\frac{2}{3}}-e \phi(x) \\
& \kappa_{s}(x)=k T \ln \left[\frac{n_{s}(x)}{N c}\right]+E_{c}-e \phi(x)
\end{aligned}
$$

where $\hbar, m, k, T$, and $E_{c}$ denote the reduced Planck constant, the effective electron mass, the Boltzmann constant, the temperature, and the bottom of conduction band, respectively. The effective density of states $N_{c}$ is expressed as $N c=2\left(\frac{m_{s} k T}{2 \pi \hbar^{2}}\right)^{3 / 2}$, where $m_{s}$ represents the effective mass of electrons in ferroelectrics.

A simple approach is employed that the polarization is treated as an infinite thin sheet of charges located at the interfaces. Thus, the boundary condition for the electrostatic potentials can be expressed as [38]:

$$
\begin{aligned}
& \left.\varepsilon_{s} \varepsilon_{0} \frac{d \phi(x)}{d x}\right|_{X_{l}^{+}}-\left.\varepsilon_{m} \varepsilon_{0} \frac{d \phi(x)}{d x}\right|_{X_{l}^{-}}=-\sigma\left(X_{l}\right)=P \\
& \left.\varepsilon_{m} \varepsilon_{0} \frac{d \phi(x)}{d x}\right|_{X_{r}^{+}}-\left.\varepsilon_{s} \varepsilon_{0} \frac{d \phi(x)}{d x}\right|_{X_{r}^{-}}=-\sigma\left(X_{r}\right)=-P
\end{aligned}
$$

where $\sigma, P, X_{l}$ and $X_{\gamma}$ denote the interface charge density, the ferroelectric polarization, the position of left interface, and the position of right interface, respectively. A simple boundary condition is used to solve the continuity equation $[36,37]$ :

$$
\kappa(x)=\text { continuous }
$$

These equations (1)-(6) coupled with boundary conditions (7)-(9) can be solved using the iterative method.
The model was applied to the experimental data in $\mathrm{SRO} / \mathrm{BFO} / \mathrm{Pt}$ structures $[26,27]$. For BFO, the saturation polarization is about $65 \mu \mathrm{C} / \mathrm{cm}^{2}[26,27]$. Other parameters are listed in Table 1 . The theoretical results agree well with experimental data as shown in Fig. $2[26,28]$, showing that this model can well describe the switchable diode effect. Based on the model, it was theoretically revealed that the ferroelectric polarization could modulate the barrier at the metal/ferroelectrics interfaces, and the Schottky-to-Ohmic conversion can occur under a large enough electric field [30]. Consequently, the polarity of the ferroelectric diode could be switched with the polarization reversal, giving rise to the switchable diode effect in ferroelectric diodes. Therefore, the ferroelectric polarization dominates the switchable diode effect.

Furthermore, this theoretical model can well explain the effect of the electrodes in ferroelectric diodes. The Schottky barrier for an ideal Schottky diode is determined by the difference of the metal work function and the semiconductor electron affinity, thus the work function of the electrodes can influence charge transport and the switchable diode effect in ferroelectric diodes. In order to further understand the effect of electrodes on the transport characteristics in ferroelectric diodes, we calculated the barrier height variation, defined as the change of the barrier height with and without a polarization, as a function of the polarization in SRO/BFO/Pt. From Fig. 3 [30], $\Delta \phi_{P t / B F O}$ as a function of the polarization deviates away from the linear dependence in the case of a large positive polarization (pointing from $\mathrm{SRO}$ to $\mathrm{Pt}$ ), attributed to a high electron density $2.0 \times 10^{20} \mathrm{~cm}^{-3}$ under a polarization of $150 \mu \mathrm{C} / \mathrm{cm}^{2}$. However, $\Delta \phi_{S R O / B F O}$ does not show this deviation in the case of a large negative polarization (pointing from $\mathrm{Pt}$ to $\mathrm{SRO}$ ), because the electron density is $2.1 \times 10^{17} \mathrm{~cm}^{-3}$ under a polarization of $-150 \mu \mathrm{C} / \mathrm{cm}^{2}$. In addition, $\left|\Delta \phi_{P t / B F O}\right|$ is larger than $\left|\Delta \phi_{S R O / B F O}\right|$ under the same polarization, mainly due to a smaller permittivity of Pt. Hence, we had better select electrodes with a smaller permittivity besides a suitable work function, such as noble metals rather than oxide electrodes, to obtain a more pronounced switchable diode effect.

Table 1 The parameters used in calculations (Reprinted with permission from Ref. [30]. Copyright 2011, American Institute of Physics)

\begin{tabular}{lccc}
\hline & $\mathrm{SrRuO}_{3}$ & $\mathrm{Pt}$ & $\mathrm{BiFeO}_{3}$ \\
\hline Dielectric constant $\left(\varepsilon_{0}\right)$ & 8 & 2 & 100 \\
Mobility $\left(\mathrm{cm}^{2} /(\mathrm{V} \cdot \mathrm{s})\right)$ & 10 & 60 & 5 \\
Effective mass $\left(\mathrm{m}_{0}\right)$ & 5 & 1 & 5 \\
Concentration $\left(\mathrm{cm}^{-3}\right)$ & $1.2 \times 10^{22}$ & $1.5 \times 10^{22}$ & $4 \times 10^{16}$ \\
Work function $(\mathrm{eV})$ & 5.0 & 5.55 & 4.7 \\
\hline
\end{tabular}




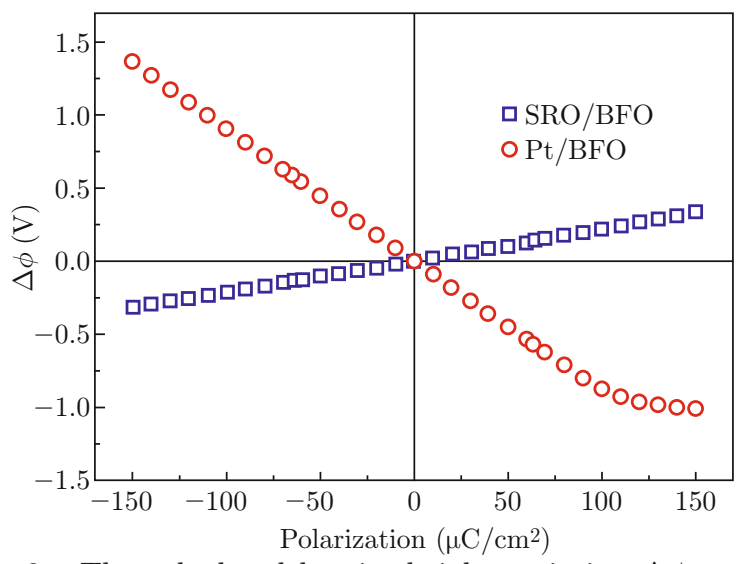

Fig. 3 The calculated barrier height variation $\Delta \phi$ versus the polarization in the $\mathrm{SRO} / \mathrm{BFO} / \mathrm{Pt}$ structure. (Reprinted with permission from Ref. [30]. Copyright 2011, American Institute of Physics)

\section{Conclusions}

In conclusion, we have reviewed recent progress in the switchable diode effect in both experimental and theoretical aspects. Many recent investigations confirmed that the barrier at the metal/ferroelectrics interfaces could be modulated by the polarization charges and the ferroelectric polarization that can be reversed by an external electric field plays a dominant role in the switchable diode effect. The switchable ferroelectric control of the diode polarity may not be realized if the oxygen vacancies layer is formed at the interfaces in ferroelectric diodes, and the switchable diode effect could be obtained after removing the excess oxygen vacancies. In theoretical aspect, we discuss a self-consistent numerical model that can well describe the switchable diode effect in MFM structures. Calculated results further predict that it is a better choice to select metals with a smaller permittivity, such as noble metals, to obtain a more pronounced switchable diode effect. It is expected that these recent experimental and theoretical works can be of great value for the further progress of the designing in ferroelectric memory devices and a deep understanding of the underlying physics in ferroelectric diodes.

Although considerable progress has been achieved in recent years in the ferroelectric diodes, much more can be expected in future. With the decrease of the ferroelectric film down to tens of nanometers, novel phenomena could emerge due to the competition between the tunneling and the drift-diffusion or thermionic emission in MFM structures. Ferroelectric materials show a rather large photovoltage exceeding several times the band gap, which could be applied in robust optoelectronic devices [22,39-41]. BFO, a typical multiferroic material, offers opportunities for us to control the states of novel devices both electrically and magnetically [42-
44]. Besides, the coupling among the ferroelectric, ferromagnetic, and optical properties provides a new route to design multifunctional devices. In terms of the theoretical aspect, it would be a more powerful method to analyze charge transport in MFM structures by considering the multi-domain structure, the hole transport process, the spatial distribution of the polarization charges.

\section{Acknowledgements}

This work was supported by the National Basic Research Program of China (Nos. 2012CB921403 and 2013CB328706) and the National Natural Science Foundation of China (Nos. 11134012 and 11174355).

\section{References}

[1] J. F. Scott, "Ferroelectric memories", Springer, Heidelberg, Germany (2000).

[2] M. Dawber, K. M. Rabe and J. F. Scott, "Physics of thin-film ferroelectric oxides", Rev. Mod. Phys. 77(4), 1083-1130 (2005). http://dx.doi.org/10. 1103/RevModPhys. 77.1083

[3] J. F. Scott and C. A. Araujo, "Ferroelectric memories", Science 246(4936), 1400-1405 (1989). http://dx.doi. org/10.1126/science. 246.4936.1400

[4] O. Auciello, J. F. Scott and R. Ramesh, "The physics of ferroelectric memories", Phys. Today 51(7), 22-27 (1998). http://dx.doi.org/10.1063/1.882324

[5] P. Zubko and J.-M. Triscone, "Applied physics: a leak of information", Nature 460, 45-46 (2009). http://dx. doi .org/10.1038/460045a

[6] R. Meyer and R. Waser, "Hysteretic resistance concepts in ferroelectric thin films", J. Appl. Phys. 100(5), 051611-051618 (2006). http://dx.doi.org/10.1063/ 1.2337078

[7] B. C. Huang, Y. T. Chen, Y. P. Chiu, Y. C. Huang, J. C. Yang, Y. C. Chen and Y. H. Chu, "Direct observation of ferroelectric polarization-modulated band bending at oxide interfaces" Appl. Phys. Lett. 100(12), 122903-122906 (2012). http://dx.doi.org/10.1063/ 1.3691615

[8] L. Pintilie, V. Stancu, L. Trupina and I. Pintilie, "Ferroelectric schottky diode behavior from a $\mathrm{SrRuO}_{3}-$ $\mathrm{Pb}\left(\mathrm{Zr}_{0.2} \mathrm{Ti}_{0.8}\right) \mathrm{O}_{3}$-Ta structure", Phys. Rev. B 82(8), 085319-085326 (2010). http://dx.doi.org/10.1103/ PhysRevB. 82.085319

[9] E. Y. Tsymbal and H. Kohlstedt, "Applied physicstunneling across a ferroelectric" Science 313(5784), 181-183 (2006). http://dx.doi.org/10.1126/ science. 1126230

[10] M. Y. Zhuravlev, R. F. Sabirianov, S. S. Jaswal and E. Y. Tsymbal, "Giant electroresistance in ferroelectric tunnel junctions", Phys. Rev. Lett. 94(24), 246802-246805 (2005). http://dx.doi.org/10.1103/ PhysRevLett. 94.246802 
[11] V. Garcia, S. Fusil, K. Bouzehouane, S. EnouzVedrenne, N. D. Mathur, A. Barthélémy and M. Bibes, "Giant tunnel electroresistance for non-destructive readout of ferroelectric states", Nature 460(7251), 8184 (2009). http://dx.doi.org/10.1038/nature08128

[12] P. Maksymovych, S. Jesse, P. Yu, R. Ramesh, A. P. Baddorf and S. V. Kalinin, "Polarization control of electron tunneling into ferroelectric surfaces", Science324(5933), 1421-1425 (2009). http://dx.doi. org/10.1126/science. 1171200

[13] V. Garcia, M. Bibes1, L. Bocher, S. Valencia, F. Kronast, A. Crassous, X. Moya, S. Enouz-Vedrenne, A. Gloter, D. Imhoff, C. Deranlot, N. D. Mathur, S. Fusil, K. Bouzehouane and A. Barthélémy, "Ferroelectric control of spin polarization", Science 327(5969), 11061110 (2010). http://dx.doi.org/10.1126/science. 1184028

[14] P. W. M. Blom, R. M. Wolf, J. F. M. Cillessen and M. P. C. M. Krijn, "Ferroelectric schottky diode", Phys. Rev. Lett. 73(15), 2107-2110 (1994). http://dx.doi. org/10.1103/PhysRevLett.73.2107

[15] Y. Watanabe, "Reproducible memory effect in the leakage current of epitaxial ferroelectric/conductive perovskite heterostructures", Appl. Phys. Lett. 66(1), 28-30 (1995). http://dx.doi.org/10.1063/1.114170

[16] P. V. D. Sluis, "Non-volatile memory cells based on $\mathrm{Zn}_{x} \mathrm{Cd}_{1-x} \mathrm{~S}$ ferroelectric schottky diodes", Appl. Phys. Lett. 82(23), 4089-4091 (2003). http://dx.doi.org/ 10.1063/1.1581365

[17] G. Z. Liu, K. J. Jin, J. Qiu, M. He, H. B. Lu, J. Xing, Y. L. Zhou and G. Z. Yang, "Resistance switching in $\mathrm{BaTiO}_{3-\delta} / \mathrm{Si} p-n$ heterostructure", Appl. Phys. Lett. 91(25), 252110-252113 (2007). http://dx.doi. org/10.1063/1.2821369

[18] S. Y. Yang, L. W. Martin, S. J. Byrnes, T. E. Conry, S. R. Basu, D. Paran, L. Reichertz, J. Ihlefeld, C. Adamo, A. Melville, Y.-H. Chu, C.-H. Yang, J. L. Musfeldt, D. G. Schlom, J. W. Ager and R. Ramesh, "Photovoltaic effects in $\mathrm{BiFeO}_{3}$ ", Appl. Phys. Lett. 95(6), 062909-062911 (2009). http://dx.doi.org/10.1063/ 1.3204695

[19] Y. Shuai, S. Q. Zhou, C. G. Wu, W. L. Zhang, D. Bürger, S. Slesazeck, T. Mikolajick, M. Helm and H. Schmidt, "Memristor behaviors of highly oriented anatase $\mathrm{TiO}_{2}$ film sandwiched between top Pt and bottom $\mathrm{SrRuO}_{3}$ electrodes", Appl. Phys. Express 4(4), 041101-041103 (2011). http://dx.doi.org/10.1143/ APEX . 4.041101

[20] K. Asadi, D. M. D. Leeuw, B. D. Boer and P. W. M. Blom, "Organic non-volatile memories from ferroelectric phase-separated blends", Nature Mater.7(7), 547550 (2008). http://dx.doi.org/10.1038/nmat2207

[21] K. Asadi, T. G. de Boer, P. W. M. Blom and D. M. de Leeuw, "Tunable injection barrier in organic resistive switches based on phaseseparated ferroelectric-semiconductor blends", Adv. Funct. Mater. 19(19), 3173-3178 (2009). http://dx. doi.org/10.1002/adfm. 200900383

[22] T. Choi, S. Lee, Y. J. Choi, V. Kiryukhin and S.-W. Cheong, "Switchable ferroelectric diode and photovoltaic effect in $\mathrm{BiFeO}_{3}$ ", Science 324(5923), 63-66 (2009). http://dx.doi.org/10.1126/science. 1168636

[23] W. D. Wu, J. R. Guest, Y. Horibe, S. Park, T. Choi, S. W. Cheong and M. Bode, "Polarization-modulated rectification at ferroelectric surfaces", Phys. Rev. Lett. 104(21), 217601-217604 (2010). http://dx.doi.org/ 10. 1103/PhysRevLett. 104.217601

[24] C. J. Won, Y. A. Park, K. D. Lee, H. Y. Ryu and N. Hur, "Diode and photocurrent effect in ferroelectric $\mathrm{BaTiO}_{3-\delta}$ ", J. Appl. Phys. 109(8), 084108-084111 (2011). http://dx.doi.org/10.1063/1.3569619

[25] C. H. Yang, J. Seidel, S. Y. Kim, P. B. Rossen, P. Yu, M. Gajek, Y. H. Chu, L. W. Martin, M. B. Holcomb, Q. He, P. Maksymovych, N. Balke, S. V. Kalinin, A. P. Baddorf, S. R. Basu, M. L. Scullin and R. Ramesh, "Electric modulation of conduction in multiferroic Cadoped $\mathrm{BiFeO}_{3}$ films", Nature Mater. 8(6), 485-493 (2009). http://dx.doi.org/10.1038/nmat2432

[26] Q. Jiang, C. Wang, K. J. Jin, X. B. Liu, J. F. Scott, C. S. Hwang, T. A. Tang, H. B. Lu and G. Z. Yang, "A resistive memory in semiconducting $\mathrm{BiFeO}_{3}$ thin-film capacitors", Adv. Mater. 23(10), 1277-1281 (2011). http://dx.doi.org/10.1002/adma.201004317

[27] Wang, K. J. Jin, Z. T. Xu, L. Wang, C. Ge, H. B. Lu, H. Z. Guo, M. He and G. Z. Yang, "Switchable diode effect and ferroelectric resistive switching in epitaxial $\mathrm{BiFeO}_{3}$ thin films", Appl. Phys. Lett. 98(19), 192901-192903 (2011). http://dx.doi.org/10.1063/ 1.3589814

[28] Lee, S. H. Baek, T. H. Kim, J. G. Yoon, C. M. Folkman, C. B. Eom and T. W. Noh, "Polarity control of carrier injection at ferroelectric/metal interfaces for electrically switchable diode and photovoltaic effects", Phys. Rev. B 84(12), 125305-125313 (2011). http:// dx.doi.org/10.1103/PhysRevB.84.125305

[29] S. Hong, T. Choi, J. H. Jeon, Y. Kim, H. Lee, H. Y. Joo, I. Hwang, J. S. Kim, S. O. Kang, S. V. Kalinin and B. H. Park, "Large resistive switching in ferroelectric $\mathrm{BiFeO}_{3}$ nano-island based switchable diodes", Adv. Mater. 25(16), 2339-2343 (2013). http://dx.doi.org/ 10.1002/adma. 201204839

[30] C. Ge, K. J. Jin, C. Wang, H. B. Lu, C. Wang and G. Z. Yang, "Numerical investigation into the switchable diode effect in metal-ferroelectric-metal structures", Appl. Phys. Lett. 99(6), 063509-063511 (2011). http://dx.doi.org/10.1063/1.3624849

[31] C. Ge, K. J. Jin, C. Wang, H. B. Lu, C. Wang and G. Z. Yang, "Effect of ferroelectric parameters on ferroelectric diodes", J. Appl. Phys. 111(5), 054104-054108 (2012). http://dx.doi.org/10.1063/1.3692769

[32] G. L. Yuan and J. L. Wang, "Evidences for the depletion region induced by the polarization of ferroelectric semiconductors", Appl. Phys. Lett. 95(25), 252904-252906 (2009). http://dx.doi.org/10.1063/ 1.3268783

[33] M. F. Chisholm, W. Luo, M. P. Oxley, S. T. Pantelides and H. N. Lee, "Atomic-scale compensation phenomena at polar interfaces", Phys. Rev. Lett. 105(19), 
197602-197605 (2010). http://dx.doi.org/10.1103/ PhysRevLett.105.197602

[34] R. R. Mehta, B. D. Silverman and J. T. Jacobs, "Depolarization fields in thin ferroelectric films", J. Appl. Phys. 44(8), 3379-3385 (1973). http://dx.doi.org/ $10.1063 / 1.1662770$

[35] C. T. Black and J. J. Welser, "Electric-field penetration into metals: consequences for high-dielectricconstant capacitors", IEEE Trans. Electron Devices 46(4), 776-780 (1999). http://dx.doi.org/10.1109/ 16.753713

[36] F. Neumann, Y. A. Genenko, C. Melzer and H. von Seggern, "Self-consistent theory of unipolar chargecarrier injection in metal/insulator/metal systems", J. Appl. Phys. 100(8), 084511-084518 (2006). http://dx . doi.org/10.1063/1.2360383

[37] F. Neumann, Y. A. Genenko, C. Melzer, S. V. Yampolskii and H. von Seggern, "Self-consistent analytical solution of a problem of charge-carrier injection at a conductor/insulator interface", Phys. Rev. B 75(20), 205322-205331 (2007). http://dx.doi.org/10.1103/ PhysRevB. 75. 205322

[38] D. Schroeder, "Modelling of interface carrier transport for device simulation", Springer-Verlag, Berlin, Germany (1994). http://dx.doi.org/10.1007/ 978-3-7091-6644-4

[39] S. Y. Yang, J. Seidel, S. J. Byrnes, P. Shafer, C. H. Yang, M. D. Rossell, P. Yu, Y. H. Chu, J. F. Scott,
J. W. Ager, L. W. Martin and R. Ramesh, "Abovebandgap voltages from ferroelectric photovoltaic devices", Natue Nanotech. 5(2), 143-147 (2010). http:// dx.doi.org/10.1038/nnano. 2009.451

[40] W. Ji, K. Yao and Y. C. Liang, "Bulk photovoltaic effect at visible wavelength in epitaxial ferroelectric $\mathrm{BiFeO}_{3}$ thin films", Adv. Mater. 22(15), 1763-1766 (2010). http://dx.doi.org/10. 1002/adma. 200902985

[41] L. Wang, Y. L. Jin, K. J. Jin, C. Wang, H. B. Lu, C. Wang, C. Ge, X. Y. Chen, E. J. Guo and G. Z. Yang, "Photo-resistance and photo-voltage in epitaxial $\mathrm{BiFeO}_{3}$ thin films", Europhys. Lett. 96(1), 17008 (2011). http://dx.doi.org/10.1209/0295-5075/96/ 17008

[42] N. A. Spaldin, S. W. Cheong and R. Ramesh, "Multiferroics: past, present, and future", Phys. Today 63(10), 38-43 (2010). http://dx.doi.org/10.1063/1. 3502547

[43] G. Catalan and J. F. Scott, "Physics and applications of bismuth ferrite", Adv. Mater. 21(24), 2463-2485 (2009). http://dx.doi.org/10.1002/ adma. 200802849

[44] J. Ma, J. M. Hu, Z. Li and C. W. Nan, "Recent progress in multiferroic magnetoelectric composites: from bulk to thin films", Adv. Mater. 23(9), 1062-1087 (2011). http://dx.doi.org/10. 1002 /adma. 201003636 